\title{
RETRACTED ARTICLE: Local Well-Posedness for the Incompressible Full Magneto-Micropolar System with Vacuum
}

\author{
Jishan Fan $^{1} \cdot$ Zhaoyun Zhang ${ }^{2} \cdot$ Yong Zhou ${ }^{2,3}$ \\ Published online: 17 October 2019 \\ ๑) Malaysian Mathematical Sciences Society and Penerbit Universiti Sains Malaysia 2019
}

The authors have retracted this article [1] because it was published in error. The correct manuscript has been published as [2]. The authors and Springer Nature apologize to readers. The online version of this article contains the full text of the retracted article as electronic supplementary material.

\section{References}

1. Fan, J., Zhang, Z., Zhou, Y.: Local well-posedness for the incompressible full magneto-micropolar system with vacuum. Bull. Malays. Math. Sci. Soc. (2019). https://doi.org/10.1007/s40840-019-008505

2. Fan, J., Zhang, Z., Zhou, Y.: Regularity criteria for a Ginzburg-Landau-Navier-Stokes in a bounded domain. Bull. Malays. Math. Sci. Soc. (2019). https://doi.org/10.1007/s40840-019-00866-X

Publisher's Note Springer Nature remains neutral with regard to jurisdictional claims in published maps and institutional affiliations.

Electronic supplementary material The online version of this article (https://doi.org/10.1007/s40840019-00850-5) contains supplementary material, which is available to authorized users.

$\bowtie$ Yong Zhou

zhouyong3@mail.sysu.edu.cn

Jishan Fan

fanjishan@njfu.edu.cn

Zhaoyun Zhang

zhangzhy93@mail2.sysu.edu.cn

1 Department of Applied Mathematics, Nanjing Forestry University, Nanjing 210037, People's Republic of China

2 School of Mathematics (Zhuhai), Sun Yat-sen University, Zhuhai 519082, Guangdong, People's Republic of China

3 Department of Mathematics, Zhejiang Normal University, Jinhua 321004, Zhejiang, People's Republic of China 